\title{
A Computer Simulation of Single Fiber Pull Out Process in a Composite
}

\author{
WEN ZHONG AND NING PAN* \\ Division of Textiles and Clothing \\ Department of Biological and Agricultural Engineering \\ University of California, Davis, CA 95616, USA \\ (Received January 27, 2003) \\ (Revised April 14, 2003)
}

\begin{abstract}
A stochastic approach, the Ising model combined with Monte Carlo simulation, is employed to study the process of a fiber debonding and being pulled out from a fiber reinforced composite. The complicated mechanisms involved can be realistically simulated more than ever with a simple algorithm that is applicable to such phenomena as partial debonding, fiber breaking and matrix yielding, all of which are difficult to deal with by existing methods. The important factors influencing the debonding and the pull out behavior can be represented by corresponding coefficients in an energy expression of the system. The results of parametric studies are in good qualitative agreement with the behavior of real systems, indicating the power and usefulness of this approach in the studies of many interfacial phenomena. Finally, application of this method to simulate the fiber peel process is also demonstrated.
\end{abstract}

KEY WORDS: Ising model, Monte Carlo simulation, Debonding, Interface, Composites, Fiber pull out.

\section{INTRODUCTION}

\begin{abstract}
A S THE USE of advanced fiber reinforced composites is extended to various engineering applications, extensive research has been carried out to further understand the mechanical properties of these materials so as to benefit new design and applications.

It has become a common knowledge that the mechanical properties of fiber reinforced composites are dependent on the specific properties of the matrix and reinforcing fibers and, just as importantly if not more so, their interfacial properties, which ensure stress transfer between matrix and fibers. In fact, study of the mechanisms of the matrix-fiber interactions via the interface is now one of a few key issues in composite studies.

Several experimental techniques have been developed to characterize the interfacial properties in a fiber reinforced composite. These include the single fiber pull out test, single fiber fragmentation test, fiber peel test and micro-indentation test. Among them, the
\end{abstract}

*Author to whom correspondence should be addressed. E-mail: npan@ucdavis.edu 
pull out test seems to be the most direct approach in studying composite interfacial behavior.

In a pull out test, a single fiber is embedded in a block of matrix (usually an epoxy resin). The fiber is then pulled out from the cured resin by applying a tensile load on the fiber. The process of having the fiber pulled out from the matrix is observed to demonstrate several typical stages; fiber debonding, postdebonding friction of fiber against debonded surfaces, and finally, either the fiber breaking or matrix being pulled out by the fiber.

To better interpret the experimental results, numerous theoretical models have been developed for describing the pull out test, still the theoretical analysis of this seemingly simple test method remains to be a challenging problem. Generally, these analyses can be classified into two categories [1]. In the approach based on shear strength criterion, debonding occurs when the interface shear stress reaches the interface shear bond strength. In the energy-based approach, extension of a debond crack requires the potential energy release rate of the composite constituents to reach a critical value, i.e., the interface fracture toughness.

In the strength approach where the shear strength criterion is used, the shear stress distribution along the interface should be evaluated. Greszczuk [2] first modified Cox's shear-lag model [3] to derive the shear stress distribution along the fiber length under the assumptions of elastic matrix and complete debonding along the interface. Since then, more work has been established based on this shear-lag model [4]. Lawrence et al. [5] introduced the concept of a partial debonding between fiber and matrix where debonding is initiated at the entrance point of fiber to matrix, and propagate to the end of the fiber. Consequently, the effect of frictional shear stress during debonding process is included. The above models assume that the fibers are embedded in a semi-infinite matrix so as to ignore the deformation of the matrix itself; whereas Hsueh [6] considered a shear-lag model where a fiber is located at the center of a coaxial cylindrical shell of matrix so that the effect of shear deformation in the matrix is thereby included.

In spite of the tremendous efforts to validate these theoretical models, a closed analytical solution with reasonable satisfaction is still not available. Although the Finite Element Method (FEM) provides a more precise tool to solve the models numerically, such nonlinear factors as the interfacial friction and matrix inelasticity have not been fully accounted for in the FEM analysis, mainly because a nonlinear analysis requires a dramatically increased computational expenditure [7,8].

The energy approach includes the early work of Outwarter and Murphy [9]. More recently, Stang and Shah [10] derived a debonded criterion based on a compliance analysis, stating that the energy approach to the debonding problem is preferable to the maximum shear stress criterion. Gao et al. [11] included friction in the debonding criterion, where a prebonded interface was modeled as a crack propagating stably along the interface with a constant interfacial fracture toughness. Gao's model was later applied by Kim et al. [12] to determine the inherent interfacial properties including interfacial fracture toughness, coefficient of friction and the residual fiber clamping stress. The energy-based models of the pull out process can also be solved with the aid of FEM [13].

When comparisons are made between the strength-based and the energy approaches, it is generally agreed that the strength theory neglecting friction can be applied to a totally unstable debonding process with short embedded fiber lengths, whereas the energy method can describe stable debonding usually with long embedded fiber lengths [12,14]. But there is still no acceptable model for describing the more frequent behavior of the partial debonding [14]. 
In this paper, a statistical method, the Ising model combined with Monte Carlo simulation, is used to study the process of debonding and the final pull out of a fiber from a matrix in various cases. The Ising model was first presented by Ising to study the phenomenon of ferromagnetic phase transition [15]. Since then it has been frequently used to study a system that consists of interactive subsystems, each of which bearing two interchangeable states [16-18].

This paper represents a new effort to apply the Ising model and Monte Carlo simulation to the description of the debonding and pull out process of a fiber from a matrix block. The target system is made from such subsystems as fiber and matrix that are interacting with each other, including both cohesion within fibers and matrix respectively, and adhesive interactions between fiber and matrix. Besides the pull out curves, the entire debonding, and final pull out process as the results of such interactions are displayed graphically, and therefore a direct view of such phenomena as partial debonding, fiber breaking and matrix failure during the pull out process is demonstrated, which was absent in previous models. At the end, the application of this method to simulate successfully a fiber peel process is also presented.

\section{DESCRIPTION OF THE ISING MODEL}

In the field of statistical thermodynamics, the macro characteristics of a system are always the reflection of the interactions and the resulting balance among micro particles or cells which comprise the system. For a system composed of $N$ kinds of subsystems each with energy $e_{i}(i=1,2,3, \ldots, N)$, while the number of subsystems having the energy $e_{i}$ is $m_{i}$, the internal energy of the system is

$$
E=\sum_{i} m_{i} e_{i}
$$

where the internal energy can be altered by the change of either $m_{i}$ or $e_{i}$

$$
d E=\sum e_{i} d m_{i}+\sum m_{i} d e_{i}
$$

The first term in the right hand of Equation (2) represents the system's energy change due to the redistribution of the subsystems among the energy $e_{i}$, which is equal to the variation of the Hamiltonian $d H$. The second term represents the system's energy change due to the change of subsystems' energies. The alteration of energies of the subsystems can be thermo-dynamically driven by extrinsic work, such as the work of a tensile load and friction, which will be considered in the present work.

\section{Internal Energy of the System}

There are various methods in statistical mechanics to construct the expression of internal energy of the system, Hamiltonian, of which Ising model is chosen in the present work.

In the original Ising model, a one-dimensional system is divided into a number of lattices cells. Then the total internal energy of the system, the Hamiltonian, is the summation of 


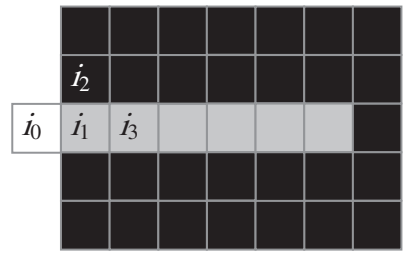

(a)

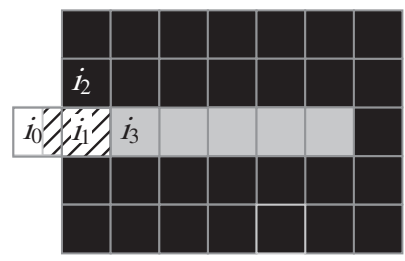

(c)

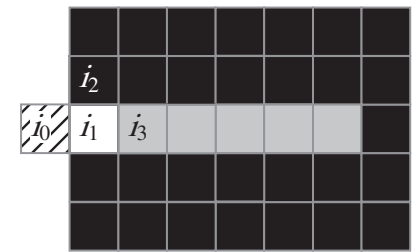

(b)

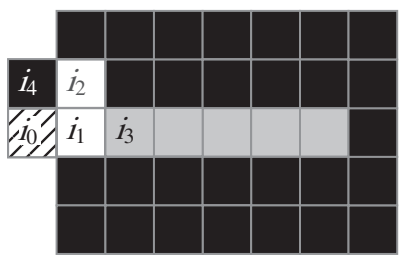

(d)
Matrix cell

Fiber cell bonded to the matrix
Debonded fiber cell

Empty cell

Figure 1. Two-dimensional Ising model in the pull out process.

the interactions between each pair of nearest neighboring cells. And the average macroscopic observable parameters of the system can be derived from the Hamiltonian via the route of statistical mechanics. The advantage of the Ising model is that, by its simple expression, it can be used to describe a complex system made of subsystems bearing two interchangeable configurations.

The target system that is studied in this paper is made of a matrix with a fiber embedded inside. In the case of a two-dimensional Ising model, the plane that comprises the fiber and matrix can be discreted into a lattice made of a set of square cells. For better explanation, a simple example with a lattice containing only $5 \times 7$ cells is shown in Figure 1(a). For convenience, the sides of a cell are made equal to the diameter of the fiber in our present case. For a pair of neighboring cells of the same medium, they are referred to as cells in connection, while for a pair of neighboring cells of different media, they are denoted as cells in contact.

To simplify the operation, the lattice is divided in such a way that each cell if not empty is filled by either fiber or matrix, that is, no fiber and matrix coexist in any single cell. As a result, four possible variables are used to describe the state of each cell:

(i) $F_{i}$ is designated to be 1 or 0 , representing a cell $i$ that is either occupied by fiber or not.

(ii) $f_{i}$ is designated to be 1 or 0 , representing a fiber cell $i$ in which fiber is either still bonded to the matrix or not.

(iii) $M_{i}$ is designated to be 1 or 0 , representing the cell $i$ that is either occupied by matrix or not.

(iv) $m_{i}$ is designated to be 1 or 0 , representing a matrix cell $i$ in which matrix is either still bonded to the bulk matrix or not (matrix failure). 
The energy of the system should be the summation of the energy of each single cell, which could be expressed as

$$
H=-A \sum_{j}^{c n} F_{i} F_{j} S_{i j}-B \sum_{j}^{c n} m_{i} m_{j} S_{i j}-G \sum_{j}^{c t}\left(f_{i} m_{j}+M_{i} f_{j}\right) S_{i j}
$$

The above equation shows that all three possible interactions in the system are considered:

(i) The cohesive energy between the connected fiber cells, as shown in the first term in the right hand of Equation (3), where $A$ represents the unit cohesion energy within fiber, $c n$ above the summation symbol denotes a summation of $F$ value over all the fiber cells in direct connection with cell $i$, and $S_{i j}$ represents the interaction area between cell $i$ and cell $j$.

(ii) The cohesive energy between the connected matrix cells, as shown in the second term, where $B$ corresponds to the unit cohesion energy within the matrix. Similarly, $c n$ above the summation symbol denotes a summation of $m$ value over all the cells in direct connection with cell $i$, and $S_{i j}$ again represents the interaction area between cell $i$ and cell $j$.

(iii) The last term represents the adhesive interactions between the contacted matrix and fiber cells, where $G$ reflects the unit adhesive energy between fiber and matrix. And $c t$ above the summation symbol denotes a summation of $m$ or $f$ values over all the cells in contact with cell $i$. When the center cell is occupied by fiber still bonded to the matrix, as in the case of computing the energy of fiber cell $i_{1}$ in Figure 1(a), only the first term in the bracket has nonzero values, while in the case of a matrix occupied the center cell, as in the case of calculating the energy of matrix cell $i_{2}$ in Figure 1(a), only the last term in the bracket has nonzero values. Equation (3) shows that $G$ is no longer in effect after debonding of the fiber cell $\left(f_{i}=0\right)$, when friction begins to work at the interface between fiber and matrix. In other words, the friction and bonding interactions between fiber and matrix never coexisted in the cell in question.

The interaction area between two cells $S_{i j}$, is determined as follows: As the two-dimensional pull out model is the projection of a real three-dimensional single fiber composite, assuming the fiber is in the shape of a perfect cylinder, the interaction area between a fiber cell $i_{1}$ and a matrix cell $i_{2}$, as shown in Figure 1(a), should be a half cylinder surface with both diameter and height equal to the fiber diameter $d$. Therefore the interaction area is $\pi d^{2} / 2$. The interaction area between fiber cell $i_{1}$ and fiber cell $i_{3}$, as shown in Figure 1(a), is a circle with diameter $d$, and hence is $\pi d^{2} / 4$. Thus

$$
S_{i j}= \begin{cases}\pi d^{2} / 2 & (\text { cell } i \text { is above or under cell } j) \\ \pi d^{2} / 4 & (\text { cell } i \text { is in connected with cell } j)\end{cases}
$$

\section{Work Done to the System by External Forces}

The work done to the system by tensile load $W_{T}$, is the product of tensile force $T$ and the elongation of the fiber. For each fiber cell of length $d$, the elongation is in the 
increment of $5 \%$ or $0.05 \mathrm{~d}$. This relatively small value may make such effects as the fiber's Poisson contraction is negligible in this study.

Next, for a single fiber cell, as friction is effective only after debonding between fiber and matrix occurs, that is, friction and adhesive interactions are mutually exclusive. The friction considered in the present paper comes only from the shrinkage stress of the matrix to the fiber, which is assumed to be constant. And as the pull out process is usually carried out in a very slow and even rate, which excluded the dynamic effect the friction energy per unit area $F_{\mathrm{r}}$ can be regarded as constant just like previous studies $[5,14]$. Therefore, the work $W_{\mathrm{F}}$ done to the system by friction is equal to the product of $F_{\mathrm{r}}$ and friction contact area. As mentioned above, friction and adhesive interaction are mutually exclusive, friction contact area should be the product of the number of the debonded yet still unbroken fiber cells $n$, and the contact area between each fiber cell and surrounding matrix $\pi d^{2}$.

\section{Monte Carlo Simulation}

Then the Hamiltonian of the system can be used in the Monte Carlo simulation to demonstrate the debonding and pull out process of a fiber from a matrix block.

In the simulation, whether a fiber cell will break/debond, or whether a matrix cell will be pulled out from the bulk matrix is determined by the Monte Carlo simulation based on the energy difference before and after the cell state changing, as described in the following. To accommodate fiber displacement during pull out, an extra cell $i_{\mathrm{o}}$ is set up as shown in Figure 1, whose initial state is $F_{\mathrm{o}}=0 . F_{\mathrm{o}}$ increases to record the fiber displacement until the completion of the pulling out test.

(i) Energy difference before and after fiber breaking.

Fiber breaking at a given cell $i$ is represented by the cell changed from a fiber occupied state $\left(F_{i}=1\right)$ to an empty state $\left(F_{i}=0\right)$, as shown in Figure $1(\mathrm{~b})$. The energy difference resulted is expressed as:

$$
\Delta E=H_{2}-H_{1}-W_{\mathrm{T}}+W_{\mathrm{F}}
$$

where $H_{2}$ and $H_{1}$ are the Hamiltonians of the system after and before the fiber breaking, $W_{\mathrm{T}}$ is the work done to the system by the tensile load, and $W_{\mathrm{F}}$ is the work done to the system by the friction. According to the discussion in the previous section, Equation (5) becomes:

$$
\Delta E=H_{2}-H_{1}-0.05 T \cdot d+\pi d^{2} n F_{\mathrm{r}}
$$

where $n$ is the number of the debonded yet still unbroken fiber cells.

(ii) Energy difference before and after fiber debonding.

A debonding fiber cell $i$ is represented by a fiber cell changed from a bonded state $\left(f_{i}=1\right)$ to a debonded state $\left(f_{i}=0\right)$, as shown in Figure 1(c). Meanwhile, the fiber cell $i_{1}$ is also elongated into the empty cell $i_{0}\left(F_{0}=0\right)$ which then changes into a partial fiber cell $\left(F_{0}=0.05\right)$ to reflect the elongation of this fiber cell during debonding. Also with the 
debonding of each succeeding fiber cell, $F_{0}$ of the partial fiber cell is accrued with a value of 0.05 each. This energy difference can also be calculated using Equations (3) and (6).

(iii) The energy difference before and after the matrix cell pull out.

Matrix in cell $i$ pulled out can be represented by a cell changing from a matrix occupied state $\left(M_{i}=1, m_{i}=1\right)$ to an empty state $\left(M_{i}=0, m_{i}=0\right)$, as shown in Figure $1(\mathrm{~d})$. The energy difference is expressed as

$$
\Delta E=H_{2}-H_{1}-W_{\mathrm{F}}
$$

With the total energy difference for this arbitrary cell $i$, the probability $W$ for a change of the system from a state with the energy $E_{1}$ to a state with the energy $E_{2}$ is calculated according to the Metropolis function [15]:

$$
W\left(E_{2} \mid E_{1}\right)=\min [1, \exp (-\Delta E / \beta)], \quad(0<W \leq 1)
$$

where $W=1$ when $\Delta E=E_{2}-E_{1} \leq 0$, or $E_{2} \leq E_{1}$, that is, a change from a higher energy state $E_{1}$ to a lower state $E_{2}$ is always favored. Otherwise, $0<W<1$ or $E_{2}>E_{1}$. In this case a number $r$ uniformly distributed between 0 and 1 is randomly selected, the state change takes place when $r<W$. Obviously, the internal energy difference $\Delta E$ for either fiber debonding, fiber breaking or matrix pull out is positive, making these failure behaviors quite unlikely to happen spontaneously. Only with certain amount of work from external force, which counteracts the positive internal energy difference, could the probability $W$ be sufficient to start the change of states.

$\beta$ is a thermodynamics constant, reflecting the effect of thermal perturbation, and is proportional to the absolute temperature. For all the simulations in the present study, $\beta$ is given a small value 0.008 , since in room temperature where the pull out tests are usually carried out, the thermal energy contribution to the system due to thermal fluctuations is negligible.

The flow chart for the whole simulation program is shown in Figure 2. In the present study, the realistic macro system is regarded as a discretisized assembly composed of micro cells with interactions. Accordingly, the failure process of the bulk system is the outcome of a set of failure behaviors of the constituent single cells. For each of these failure behaviors: fiber breaking, fiber debonding, and matrix yielding, the three $\Delta E s$ in Equations (5)-(7) should be examined separately for each cell.

The whole failure process starts with fiber debonding, from the entrance fiber cell to the last fiber cell embedded in the matrix in turn. For each cell, the tensile load $T$ needed to make a fiber cell debond or break is determined in a trial algorithm: First set as $0, T$ is increased steadily with a small increment of $d T(=1)$ in each iteration until either fiber debonding or breaking occurs. Then, for the same cell, whether a contacted matrix cell will be pulled out with the fiber is determined. During the process of fiber debonding, it is the adhesive interaction between the fiber and matrix that pulls the matrix cell out from the bulk matrix. Therefore, when using Equation (7) to compute the energy difference, $W_{\mathrm{F}}$ should be equal to 0 as the adhesive interaction and friction are mutually exclusive.

When the last fiber cell being debonded from the matrix, or when any of the fiber cells being broken during the debonding process, the postdebonding frictional sliding begins. During this process, only two of the failure modes, i.e., fiber breaking and matrix pull out, 


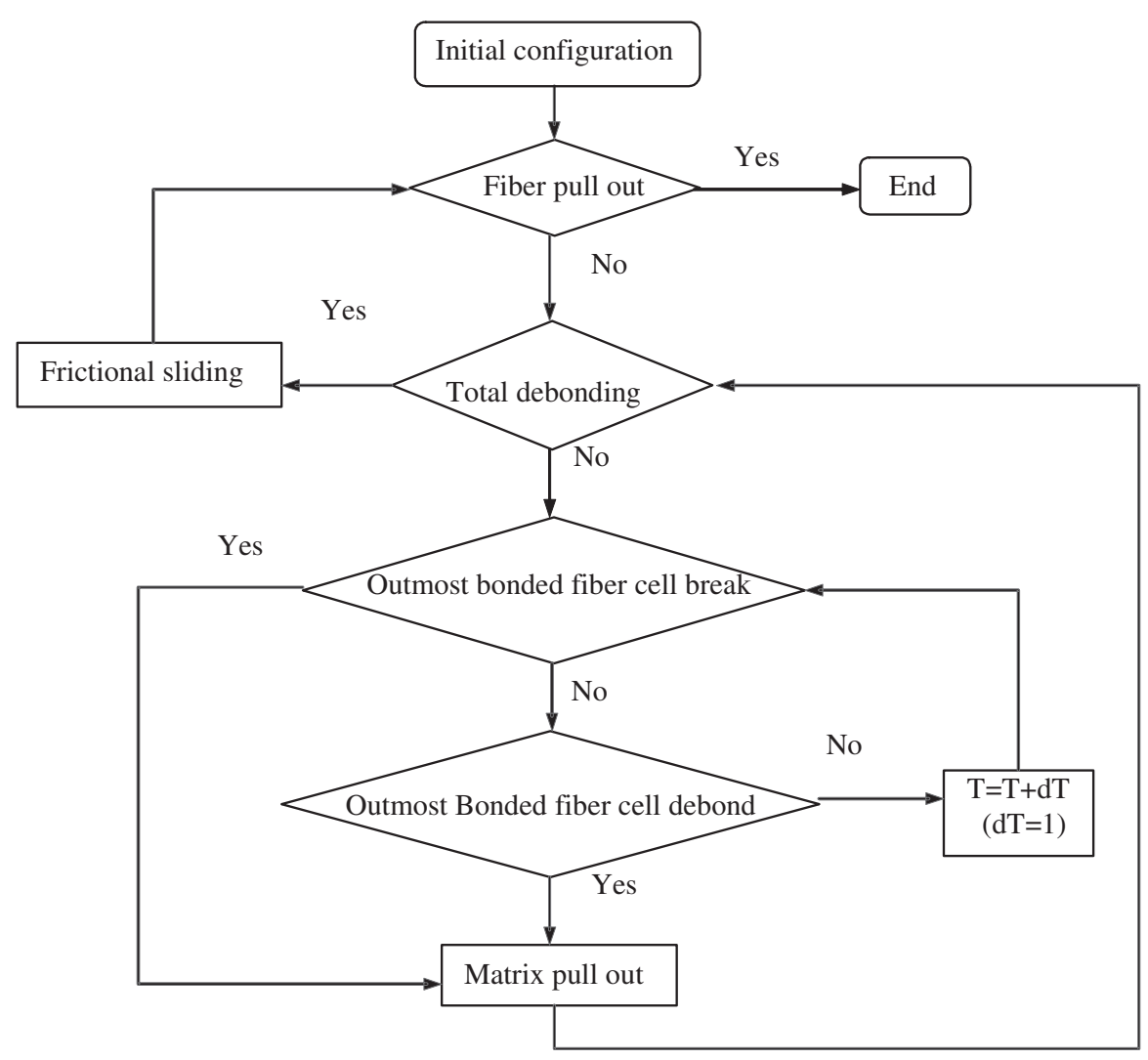

Figure 2. The flow chart of the simulation program.

are possible. Now, as the interface between fiber and matrix has totally failed, i.e., no adhesive interaction exists, $W_{\mathrm{F}}$ then takes effect, and is equal to the product of $F_{\mathrm{r}}$ and the contact area between the single matrix cell and the fiber.

\section{PARAMETRIC STUDIES}

\section{Relationship Between the System Parameters}

A computer simulation program based on the above descriptions has been developed for the pull out process of a fiber from a bulk matrix. As can be seen from Equations (3)-(7), six parameters are involved in deciding the pull-out behavior: (1) fiber cohesion coefficient $A$, unit cohesion energy between two connected fiber cells, that reflects the fiber strength, (2) matrix cohesion coefficient $B$, unit cohesion energy between two connected matrix cells, that corresponds to the matrix strength, (3) interfacial adhesive coefficient $G$, unit adhesive interaction energy between a pair of contacted fiber and matrix cells, that represents the interfacial bonding strength, (4) $F_{\mathrm{r}}$, friction energy per unit area, which denotes the friction work, (5) the embedded length of fiber in the matrix $l$, and (6) the fiber diameter $d$. As these parameters in the energy expression correspond to the characteristics 
of the materials involved, parametric studies of the pull out process can be carried out by adjusting values of these coefficients for their effects on the system behaviors.

It has to be pointed out that, for a parametric study, we usually vary one of the parameters while keeping the rest constant. A question arises from the possible interconnections among these parameters. If they are closely related, when we change the value of one parameter in question, the rest supposed to remain constant will change as well. In other words, a meaningful parametric study is possible only when all the parameters in the model are independent of, or at least very weakly dependent on, each other.

Usually, the interfacial adhesion or the bonding strength $(G)$ can be altered by various fiber surface treatments with little influence on the strengths of both fibers and matrix in the system. Also, there is little if any connection between the fiber strength $(A)$ and the matrix strength $(B)$. Either the embedded fiber length $l$ or the fiber diameter $d$ is obviously an independent parameter. Finally it has been explained above that the friction energy $F_{\mathrm{r}}$ is assumed constant, for it is caused mainly by the matrix shrinkages to the fiber. Even though the friction takes place between fiber and matrix, that does not necessarily indicate a dependence of $F_{\mathrm{r}}$ on either $A$ or $B$ which after all represent only the materials strengths. As to the relationship between $F_{\mathrm{r}}$ and $G$, they have been treated mutually exclusive as explained in the last section.

For generality, the quantitative values with corresponding dimensions of $A, B, G$, and $F_{\mathrm{r}}$ can be determined by calibrating against actual experimental tests, which will be carried out in our future work. And in the present study, all these physical parameters as well as the tensile load which were computed from the above formulations are all in relative and dimensionless terms.

And, for each combination of the values for the parameters, the seed of random numbers is set to be 0 at the beginning of the simulation so as to make the results reproducible.

\section{The Effect of Interfacial Bonding Strength on the Pull out Behavior}

Designed to measure the interfacial bonding/debonding properties of fiber reinforced composites, the pull out tests have been employed extensively to examine the influence of the interfacial adhesion or fiber-matrix bonding strength on the composite behavior.

For three sets of the total parameters with different values of $G$ while the rest remain constant as shown below, the simulated pull out behaviors are shown in Figures 4 and 5 . In Figure 4 as well as in the rest of the simulations, the lattice for each case is chosen to contain $80 \times 11$ cells.

(a) $A=500, \quad B=60, \quad G=50, \quad F_{\mathrm{r}}=6, \quad l=20 d, \quad d=0.02$

(b) $A=500, \quad B=60, \quad G=70, \quad F_{\mathrm{r}}=6, \quad l=20 d, \quad d=0.02$

(c) $A=500, \quad B=60, \quad G=130, \quad F_{\mathrm{r}}=6, \quad l=20 d, \quad d=0.02$

For the whole process of a fiber debonding and pull out from a matrix, the typical curve of the load applied versus displacement, using the parameter set in the case (a) as shown in Figure 3, comprises two parts. First there is an increase of tensile load to propagate the debonded area along the interface as well as resisting the friction at the increasing debonding area. And the required tensile load reaches the peak when the whole fiber is debonded from the matrix. The tensile load drops, and then decreases steadily when the 


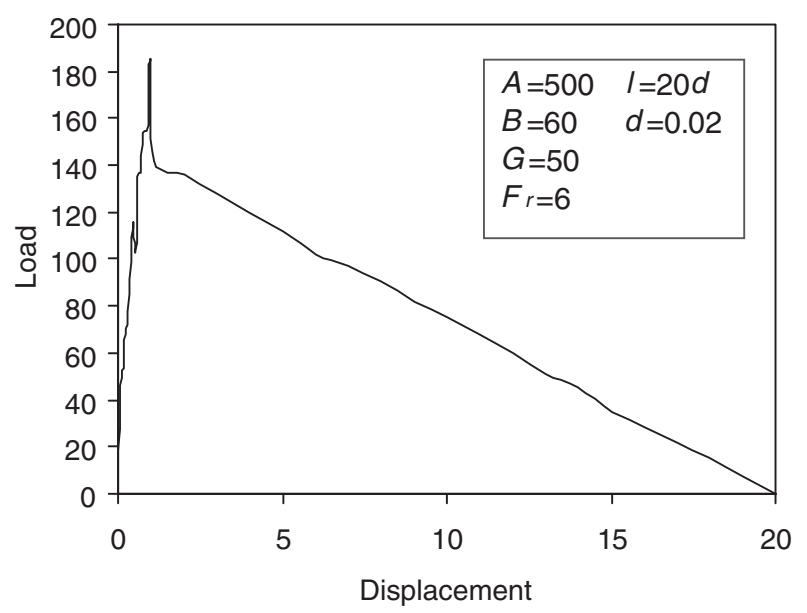

Figure 3. A typical curve of the load vs. fiber displacement during fiber debonding.

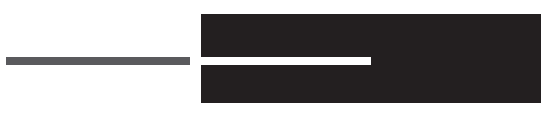

(a)

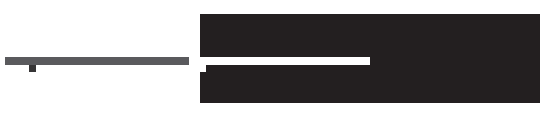

(b)

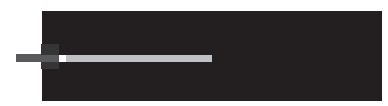

(c)

Figure 4. Pull out process for samples with different interfacial bonding strengths.

fiber is being pulled out against the friction between the fiber and matrix. The trend of the curve agrees with those results reported in existing literature [13,14]. In this and later sections, the steadily decreasing part of the curves corresponding to the postdebonding friction sliding process, is more or less truncated, so as to highlight the part of the debonding process in more details.

What's more, as a stochastic approach, the Monte Carlo simulation is capable of depicting the stochastic nature of debonding and pull out behavior in a real sample where the interface could hardly be viewed as perfect. As shown in Figure 5, the curves of the load versus displacement for pull out process do show many jerks rather than a smooth shape during debonding, which is in accordance with expectation and the reported results [14]. This reflects the irregular property distribution of the interface between fiber and matrix, justifying the use and demonstrating the power of such a stochastic approach 


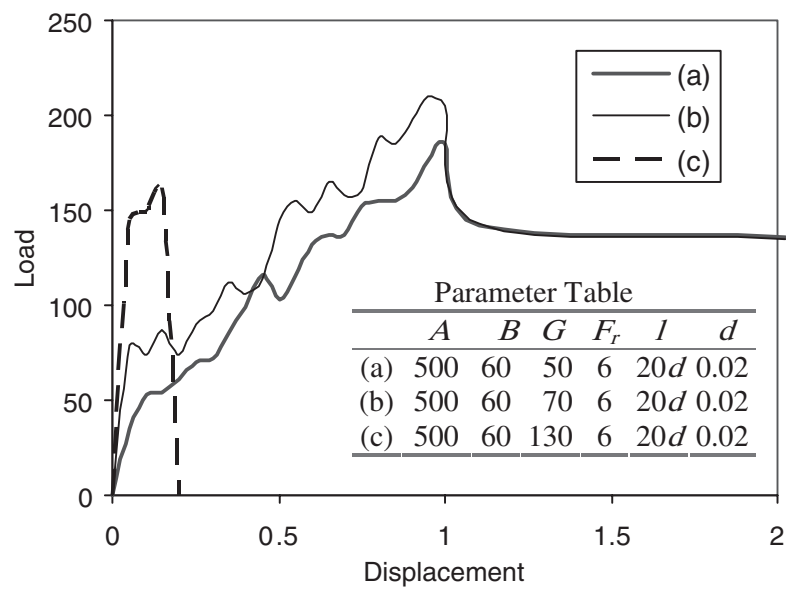

Figure 5. Simulation results of load vs. fiber displacement at various bonding strengths.

as Monte Carlo simulation in predicting polymer-fiber interfacial behavior in composite studies.

It can be seen from Figure 5 that, with the increase of the interfacial bonding strength $G$, the tensile load required to pull the fiber out increases during the debonding period. When $G$ is large enough, as shown in the case (c), the fiber breaks before debonding finishes. The rest two curves coincide in the postdebonding area as the frictions are identical in both cases.

\section{The Effect of Fiber Strength on the Pull out Behavior}

For three sets of parameters with different values of $A$ while the rest kept unchanged as shown below, the predicted pull out behaviors are shown in Figures 6 and 7.

(a) $A=500, \quad B=60, \quad G=50, \quad F_{\mathrm{r}}=6, \quad l=20 d, \quad d=0.02$

(b) $A=400, \quad B=60, \quad G=50, \quad F_{\mathrm{r}}=6, \quad l=20 d, \quad d=0.02$

(c) $A=250, \quad B=60, \quad G=50, \quad F_{\mathrm{r}}=6, \quad l=20 d, \quad d=0.02$

The curves indicate that with the decrease of fiber strength to a certain level, the fiber will break before being pulled out, as shown in Figure 7(b) and (c). And the lower the fiber strength, the earlier the break occurs, all as expected.

\section{The Effect of Matrix Strength on the Pull out Behavior}

By adjusting the values of $B$ only, the predictions of the pull out behavior are shown in Figures 8 and 9.

(a) $A=500, \quad B=60, \quad G=50, \quad F_{\mathrm{r}}=6, \quad l=20 d, \quad d=0.02$

(b) $A=500, \quad B=45, \quad G=50, \quad F_{\mathrm{r}}=6, \quad l=20 d, \quad d=0.02$

(c) $A=500, \quad B=35, \quad G=50, \quad F_{\mathrm{r}}=6, \quad l=20 d, \quad d=0.02$ 


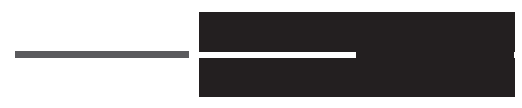

(a)

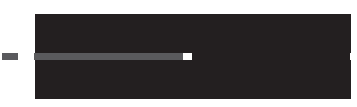

(b)

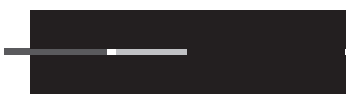

(c)

Figure 6. Simulation of pull out process for samples with different fiber strengths.

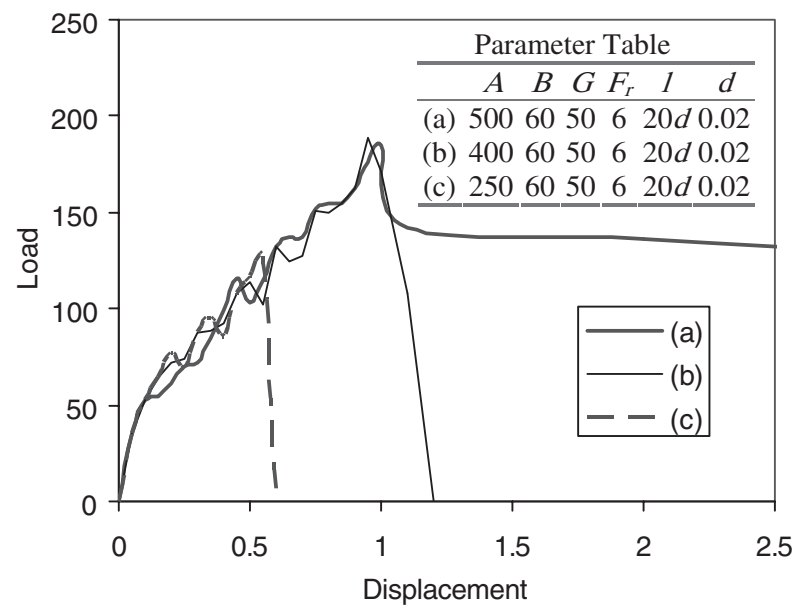

Figure 7. Simulation results of load vs. fiber displacement with different fiber strengths.

It can be seen that, with the decrease of the matrix strength, the tensile load required to pull the fiber out remains almost constant, meaning the matrix strength at this level has little effect on the pulling out load. But in the case of very low matrix strength, some fractions of the matrix are pulled out with the fiber, as a reflection of the failure of the matrix instead of debonding, since the bonding strength in this case is greater than the matrix strength itself.

\section{The Effect of Friction on the Pull out Behavior}

Further, for different values of $F_{\mathrm{r}}$, the pull out behavior is shown in Figures 10 and 11.

(a) $A=500, \quad B=60, \quad G=50, \quad F_{\mathrm{r}}=6, \quad l=20 d, \quad d=0.02$

(b) $A=500, \quad B=60, \quad G=50, \quad F_{\mathrm{r}}=9, \quad l=20 d, \quad d=0.02$

(c) $A=500, \quad B=60, \quad G=50, \quad F_{\mathrm{r}}=11, \quad l=20 d, \quad d=0.02$ 


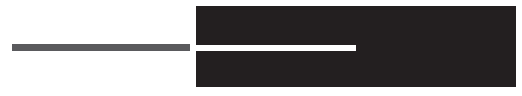

(a)

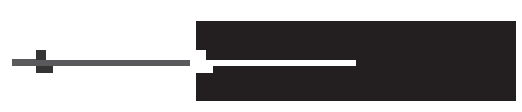

(b)

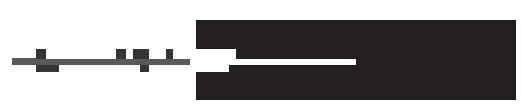

(c)

Figure 8. Simulation of pull out process for samples with different matrix strength.

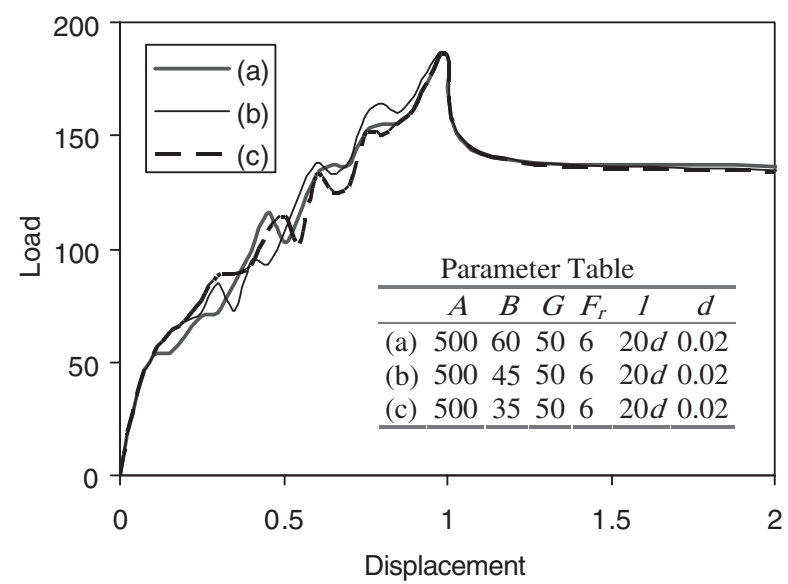

Figure 9. Simulation results of load vs. fiber displacement with different matrix strength.

The effect is that, with the increase of friction, the tensile load required to pull out the fiber and to resist the postdebonding friction increases, because for the whole system, friction works during both debonding and postdebonding processes, unlike in the case for each individual cells.

Also, the fiber breaks before being pulled out, as in the case (b), or even breaks before being totally debonded from the matrix, as in (c), if the friction is very high.

\section{The Effect of Embedded Length on the Pull out Behavior}

To study the effect of fiber embedded length $l$, three sets of parameters with different $l$ values in terms of fiber diameter $d$, the pull out behaviors are in Figures 12 and 13.

(a) $A=500, \quad B=60, \quad G=50, \quad F_{\mathrm{r}}=6, \quad l=10 d, \quad d=0.02$

(b) $A=500, \quad B=60, \quad G=50, \quad F_{\mathrm{r}}=6, \quad l=20 d, \quad d=0.02$

(c) $A=500, \quad B=60, \quad G=50, \quad F_{\mathrm{r}}=6, \quad l=30 d, \quad d=0.02$ 


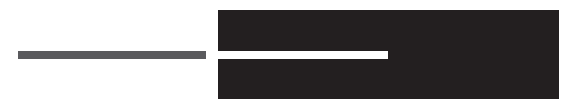

(a)

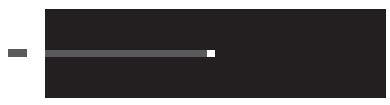

(b)

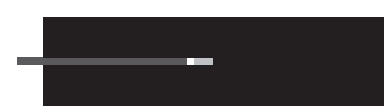

(c)

Figure 10. Simulation of pull out process for samples with different interfacial friction.

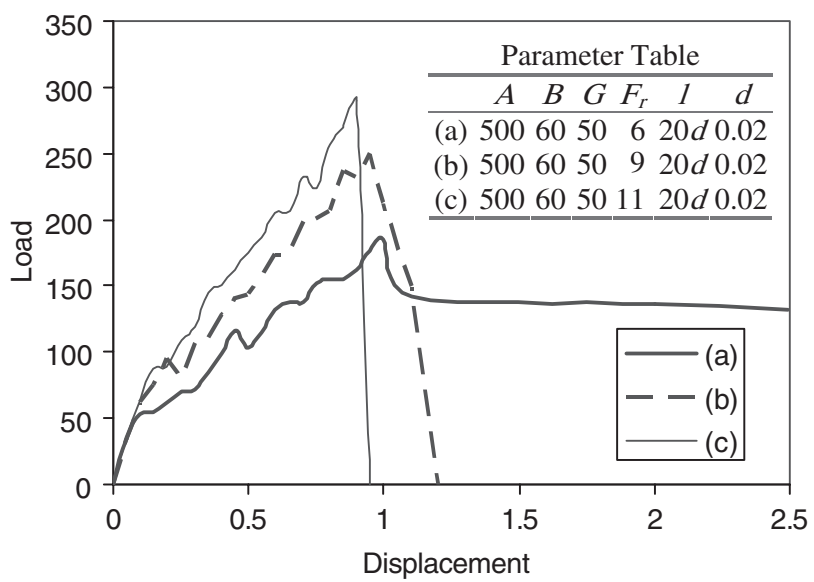

Figure 11. Simulation results of load vs. fiber displacement with different interfacial friction.

As well known, with the increase of embedded length, the tensile load required to pull the fiber out and to resist the postdebonding friction rises, due to the increase of both bonding and friction lengths, until fiber breaking before being pulled out.

\section{The Effect of Fiber Diameter on the Pull out Behavior}

The effects of fiber diameter $d$ are illustrated in Figures 14 and 15.

(a) $A=500, \quad B=60, \quad G=50, \quad F_{\mathrm{r}}=6, \quad l=20 d, \quad d=0.028$

(b) $A=500, \quad B=60, \quad G=50, \quad F_{\mathrm{r}}=6, \quad l=20 d, \quad d=0.02$

(c) $A=500, \quad B=60, \quad G=50, \quad F_{\mathrm{r}}=6, \quad l=20 d, \quad d=0.012$

It is shown that with the increase of fiber diameter, the tensile load required to pull the fiber out rises, reflecting the increase of both the bonding and friction areas between fiber 


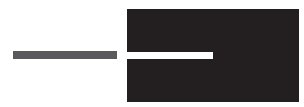

(a)

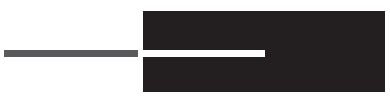

(b)

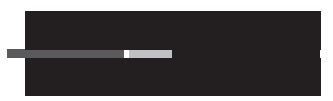

(c)

Figure 12. Simulation of pull out process for samples with different embedded lengths.

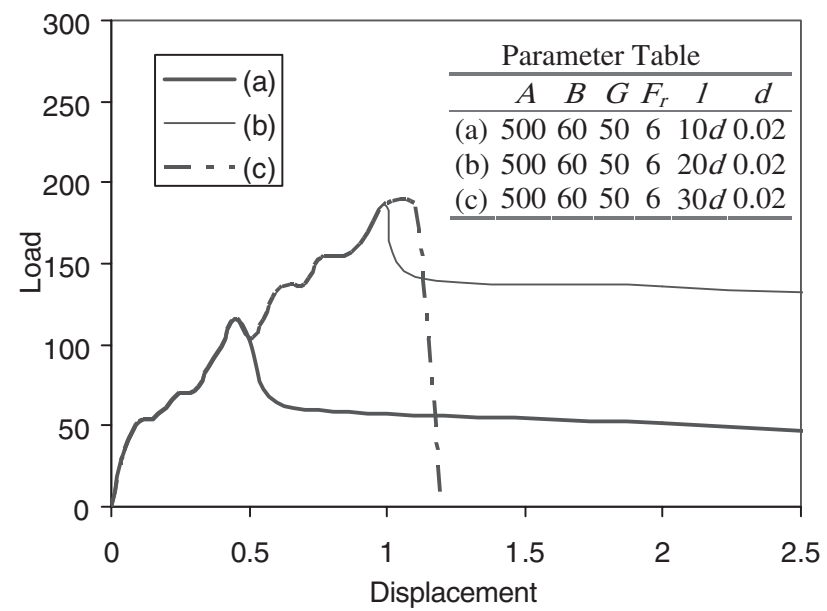

Figure 13. Simulation results of load vs. fiber displacement with different embedded lengths.

and matrix. And when the fiber diameter is very small, the fiber breaks before being pulled out because of the low loading capacity of the fiber.

From what is stated above, several advantages can be found from the present approach. First, the Ising model is capable of describing a composite system in a simple binary form, accounting for all the physical mechanisms involved, and yet generating robotically predictive results. Second, this approach is applicable to such phenomena as partial debonding, fiber breaking, and matrix failure, which are all difficult to be taken into consideration by existing models. Also, this method is applicable to other test method, such as the single fiber peel test suggested by Alimunnin and Piggott [19], as shown in the following section.

\section{THE SINGLE FIBER PEEL TEST}

Single fiber peel test is suggested by Alimunnin and Piggott [19] as a practical method to estimate the work of fracture of a fiber-polymer interface, using the curves of peel forces 


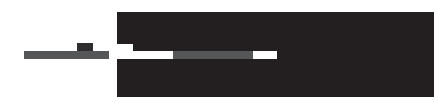

(a)

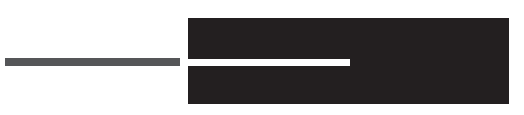

(b)

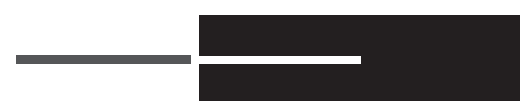

(c)

Figure 14. Simulation of pull out process with samples with different fiber diameters.

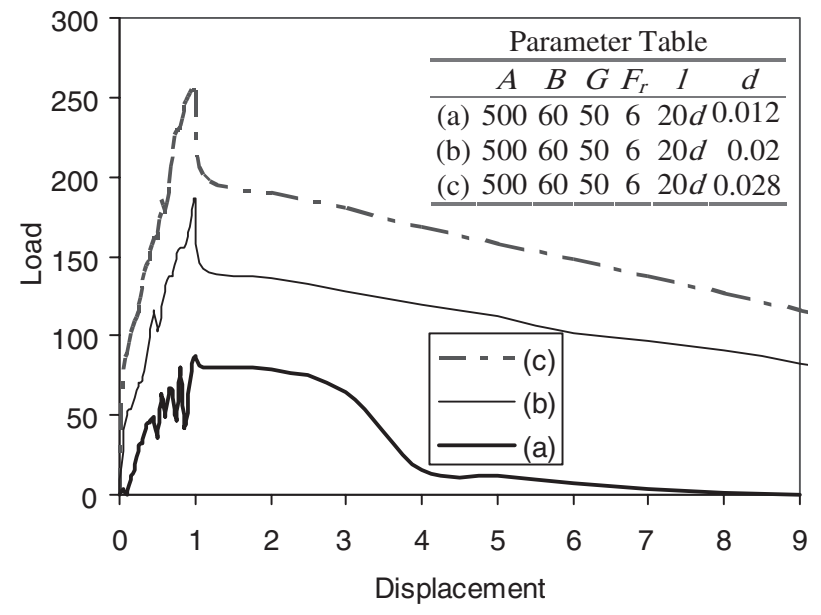

Figure 15. Simulation results of load vs. fiber displacement with different fiber diameters.

vs. displacement. In such a test, a single fiber with half of its volume embedded lengthwise in a block of cured matrix is peeled at one end off from the matrix. As polymerfiber interfacial property is the key factor in determining the peel behavior, an example of the simulation by using the present method with variation of interfacial strength $G$ is shown in Figures 16 and 17 where:

(a) $A=500, \quad B=60, \quad G=50, \quad l=40 d, \quad d=0.02$

(b) $A=500, \quad B=60, \quad G=100, \quad l=40 d, \quad d=0.02$

(c) $A=500, \quad B=60, \quad G=150, \quad l=40 d, \quad d=0.02$

It shows that with the increase of the interfacial strength $G$ while the rest coefficients are given, the required peel forces increase and the chances of matrix being pulled out by the fiber also rise. And the trend of curves in Figure17 agrees well with the experimental results reported [19]. 


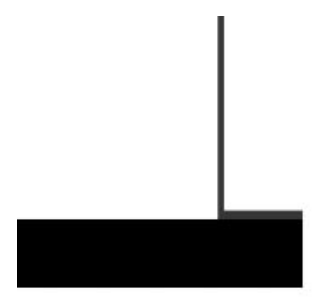

(a)

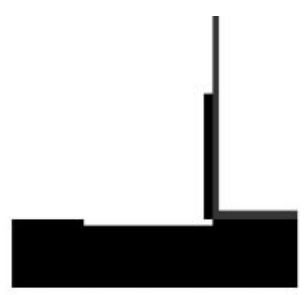

(b)

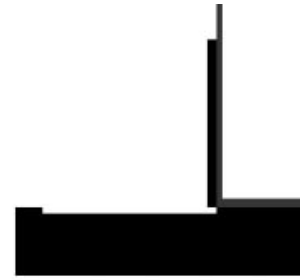

(c)

Figure 16. Simulation of single fiber peel process with different value of interfacial strengths.

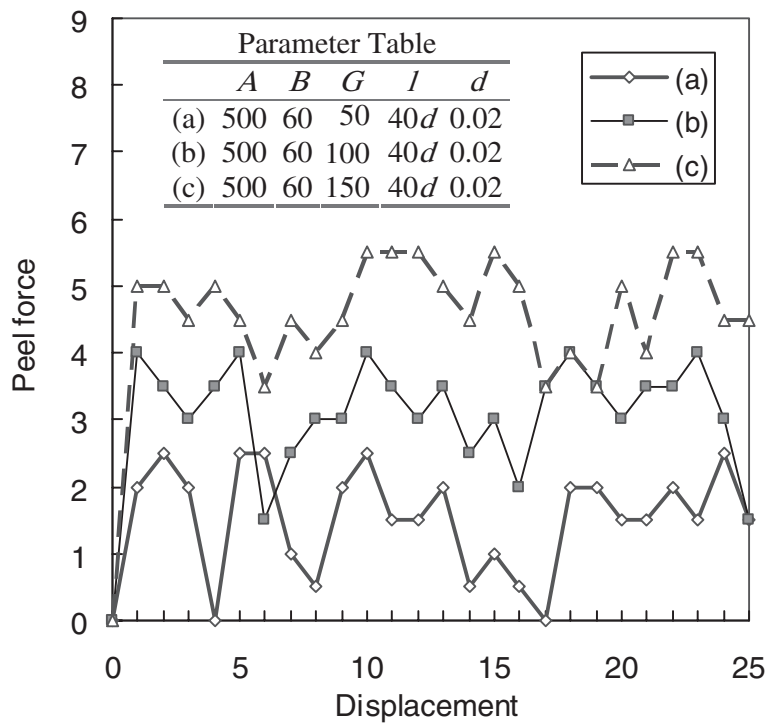

Figure 17. Peel force vs. peel out length with different value of interfacial strengths.

\section{CONCLUSIONS}

A study for applying a stochastic algorithm, the Ising model combined with the Monte Carlo method, is carried out to simulate the process of a fiber pulling out of a bulk matrix. As a result, complicated mechanisms and variables involved in this process are realistically simulated.

Several advantages of the model are demonstrated in the paper. First, the model describes a composite system in a simple approach; yet accounting for all the physical mechanisms and important parameters involved so as to realistically simulate the whole fiber pull out process. Second, this approach is applicable to such phenomena as partial debonding, fiber breaking, and matrix failure, which are all difficult to be dealt with by the existing methods. Another distinguished feature is that, this model is capable of depicting the stochastic nature of the fiber debonding and pulling out behavior in a real sample, justifying the use and demonstrating the power of such an approach in predicting the fiber-matrix interfacial characteristics in composite studies.

A series of parametric studies are carried out based on the model predictions to investigate the influences of the important parameters on the fiber pull out behavior. 
The results are in good accordance with the reported outcomes in existing literature, further validated the application of the method.

In this paper, all the physical parameters and simulated results are in relative and dimensionless terms to demonstrate the use and power of this new approach. And future work will involve the determination of these parameters by calibrating against actual experimental tests to give a direct relationship between these parameters and real materials properties.

In present study, we assume in the algorithm that the debonding process would initiate from the loaded fiber end, which is often the case in practice. Yet the model can be modified in which the present sequential scanning through the fiber-matrix interface from left to right, with one cell failing at one step, can be replaced by a more complicated scheme in which the whole interface is scanned in each step to find the failed cells wherever the locations.

Also, in the present work, a small fiber elongation increment is assumed for the pull out process in a fiber/matrix system to simplify the problem. Further studies would be performed on pull out behaviors with considerable fiber elongation so that the Poisson's effect of the fiber would be included. One of the possible ways to do so is to set the sizes of the cells as a function of computing steps instead of a constant, that is, the fiber cells deform during the pull out process, with elongation in the axial direction and contraction in the radial direction determined by the Poisson's ratio. Also a more difficult yet realistic situation may be tackled where the fiber, matrix, and even the bonding properties can be treated as statistically distributed. Finally, the approach can be applied to other failure processes of interfaces between fiber/matrix, such as the fiber peel test shown in this study, and the more complex fragmentation phenomenon.

\section{ACKNOWLEDGMENT}

Dr. Wen Zhong would like to thank the partial supports, for her work at UC Davis on this postdoctoral project, from The National Textile Center under the two grants NTC F99-D06 and NTC M02-E03.

\section{REFERENCES}

1. Kim, J.K. and Mai, Y.W. (1998). Engineered Interfaces in Fiber Reinforced Composites, Elsevier, Oxford.

2. Grezczuk, L.B. (1969). Interface in Composites, ASTM STP, 452: 42-58.

3. Cox, H.L. (1952). British Journal of Applied Physics, 3: 72-79.

4. Takaku, A. and Arridge, R.G.C. (1973). Journal of Physics, D: Applied Physics, 6: 2308-2047.

5. Lawrence, S. (1972). Journal of Materials Science, 7: 1-6.

6. Hsueh, C.H. (1990). Materials Science and Engineering, 123: 1-11.

7. Sun, W. and Lin, F. (2001). Journal of Thermoplastic Composites, 14: 327-343.

8. Beckert, W. and Lauke, B. (1997). Composites Science and Technology, 57: 1689-1706.

9. Outwarter, J.D. and Murphy, M.C. (1969). 24th Annual Tech Conf Reinforced Plastic, Paper 11C, Composites Inst. SPI, New York.

10. Stang, H. and Shah, S.P. (1986). Journal of Materials Science, 21: 953-957.

11. Gao, Y.C., Mai, Y.W. and Cotterell, B. (1988). Journal of Applied Mathematics and Physics (ZAMP), 39: 550-572. 
12. Kim, J.K., Baillie, C. and Mai, Y.W. (1992). Journal of Materials Science, 27: 3143-3154.

13. Marotzke, C. and Qiao, L. (1997). Composites Science and Technology, 57: 887-897.

14. Delfolie, C., Depecher. C. and Lefebvre, J.M. (1999). Journal of Materials Science, 34: 481-495.

15. Yeomans, J.M. (1992). Statistical Mechanics of Phase Transition, Oxford Science Publications, New York.

16. Lukas, D., Glazyrina, E. and Pan, N. (1997). Journal of Textile Institute, 88: 149-161.

17. Zhong, W., Ding, X. and Tang, Z.L. (2001). Textile Research Journal, 71: 762-766.

18. Zhong, W., Ding, X. and Tang, Z.L. (2002). Textile Research Journal, 72: 751-755.

19. Alimunnin, M.A. and Piggott, M.R. (1999). Polymer Composites, 20: 655-663. 